\title{
Classification of Colonic Polyps using Hidden Markov Models
}

\author{
M. Park ${ }^{1}$, J.S. Jesse ${ }^{1}$, R. Hofstetter ${ }^{2}$, S. Luo ${ }^{1}$, P. Summons ${ }^{1}$ \\ ${ }^{1}$ School of Design, Communication and IT, The University of Newcastle, Australia \\ ${ }^{2}$ IntelliRad Solution, East Hawthorn 3123, Victoria, Australia \\ Email: mira.park@newcastle.edu.au
}

\begin{abstract}
Colonic polyps appear as elliptical protrusions on the inner wall of the colon. Previous algorithms assumed the shape of a polyp to be a spherical cap, so these algorithms are not flexible when the polyps are various cap shapes. This paper proposes an explicit parametric model for the polyps. The model captures the overall shape of the polyp and is used to derive the probability distribution of features relevant to polyp detection. The probability distribution represents the glocal properties of the candidates (where glocal properties capture both the global information and local information of the object). A unit sphere, referred to as a brilliant sphere, is used to represent the glocal information of the polyp. The observation sequence is obtained for the polyp candidates from the brilliant sphere information, and the observation sequence is then assessed by explicit models for classification.
\end{abstract}

Keywords: Colonic polyps, Classification, Hidden Markov Models

\section{Introduction}

Colorectal cancer is the most commonly diagnosed, non-cutaneous cancer in Australia. In 2001 there were 12,844 cases of colorectal cancer $(6,961$ in men and 5,883 in women) and 4,754 deaths (2,601 in men and 2,153 in women). In Australia, the lifetime risk of developing colorectal cancer before 75 years of age is approximately one in 17 for men, and one in 26 for women [1]. With over half a million obits, colorectal cancer was ranked as the fourth leading worldwide cause of cancer death in 2002 [2]. According to the American Cancer Society [3,4,5], most colorectal cancers arise from benign colonic polyps and when tumors in the colon are found and treated early, cure rates are higher than 90 percent. Early removal of these polyps has also been shown to reduce mortality from colon cancer by $25-50 \%$ [6]. Therefore, early detection can increase survival rates.

Unfortunately one of the biggest problems with colon cancer is that most occurrences produce no symptoms in their early stage when they are small and limited to the colon. Therefore, colon cancer is an ideal disease for screening, where screening is the process of looking for a cancer in healthy persons who are at risk for the disease but have no symptoms. Optical colonoscopy is part of the standard screening protocol used to visually detect $100 \%$ of polyps and potential cancers [30]. However, screening for colon cancer lags far behind screening for other cancers, since colonoscopy is expensive, invasive and uncomfortable. The screening procedure also takes a long time and carries a risk of complications, including bleeding and perforation of the colon.

Computed Tomographic Colonography (CTC) has been shown to be a feasible approach for effective colorectal cancer screening [7] since there is no risk of bleeding or colon perforation and the performance is at least as good as optical colonoscopy [8,9]. Physicians hope it will encourage more people to be screened for colon cancer.

However, the accuracy and efficiency of viewing hundreds of CT images per examination is limited by human factors, such as attention span and eye fatigue. The review of hundreds of images for each patient is tiring and time-consuming. This is where computeraided detection (CAD) can be of help [10].

For computer-aided polyp detection (CAPD), a general algorithm consists of two phases: creating the initial polyp candidates and then a reduction of these candidates by eliminating the false positives. Previous algorithms for creating polyp candidates used the thickness of the colonic wall [11], CT attenuation and surface topology $[12,13]$, shape index and curvedness $[14,15,16]$, surface normal vector [17], curvature [18] and spherical fitting [19]. To reduce the identified candidates by eliminating false positives in the second phase, support vector machines [20], neural network classifiers, self-organizing maps, and multiobjective evolutionary techniques [21] were applied based on the features of the polyp candidates from the first phase.

The first phase of CAD systems has become more focused over the last decade and these techniques can 
now reasonably generate polyp candidates, including all true positives bigger than $6 \mathrm{~mm}$. However these methods suffer from severe false positive readings due to their inability to correctly distinguish polyps from other colonic shapes such as folds, residual materials, etc. Therefore, the second phase of CAD should be more attractive for research.

The second phase of CAD consists mainly of classification tasks, and the classifications of CAD usually adopt the algorithms mentioned before that are based on local properties such as shape index, curvedness, edge distance, and the intensity and curvature on each voxel. Such classification, based only on these local properties, produces large false positives, and overcoming this problem is the main topic addressed in this paper.

This paper focuses on the second phase (classification or reduction of false positives). Although there are many features used for classification in CAD systems, the systems are essentially based on the difference between the shape of polyps and folds. This paper presents an explicit parametric model for the various shapes of polyps. The model captures the overall shape of the polyp and is used to derive the probability distribution of features relevant for polyp detection.

In the domain being investigated polyps are regular, cup-shaped artifacts that are all protrusions from the colonic wall. The regularity in their appearance allows their variation to be well-mapped by spheroid models (shown in more detail later in Figure 2). Their variation is shown in their height from the colonic wall which influences the degree of their curvature at different heights, and their degree of roundness (circular to elliptic) at the base of the cup where it meets the colonic wall.

We use two subvolume databases of polyp candidates (which include true positives and false positives). The first phase affects the second phase substantially, but as there are no perfect colon segmentations, electronic colon cleansing, or polyp candidate generation methods, and as the classification phase depends on these results from the first phase it may not provide perfectly accurate results. Therefore, we present a novel method and this method will be evaluated by a reliable polyp candidate database.

\section{Survey of Prior Work}

The literature proposes a large number of CAD algorithms for polyp detection. The work of Vining et al. [11] was the earliest attempt at automated polyp detection. They used the thickness of colonic wall and detected regions with abnormal wall thickness as polyps. Their method achieved 73\% sensitivity with 9 to 90 false positives per dataset (FP/dataset). Yoshida and Nappi. [14] identified candidates using the shape index of each voxel, curvedness, directional gradient concentration, and quadratic discriminant analysis of small volumes of interest. They classified the candidates using fuzzy clustering to distinguish polyps from healthy tissue. They reported $89 \%$ sensitivity with $2 \mathrm{FP} /$ dataset. Kiss et al. [19] combined the intersection of surface normals and spherical fitting to detect polyps. They produced $85 \%$ sensitivity for polyps larger than $6 \mathrm{~mm}$ with 2.82 FP/dataset. They also found that surface curvature methods have a small number of false-positive findings, but at the same time have a very low polyp detection rate. On the other hand, the surface normal method has a high detection rate, while generating a lot of false-positive findings. Paik et al. [17] proposed the surface normal overlap method which evaluated the local convexity of the colonic wall using the normal intersection. They suggested that the normals at the surface intersect in a concentrated area within the hemispherical polyp. Summer et al. [12,13] used the mean, the Gaussian and the principal curvatures, and the sphericity ratio to identify polyp candidates. The sensitivity of their technique varied between $29 \%$ and $100 \%$, with 6 to 20 false positives per dataset. Huang et al. [21] computed curvatures by fitting cubic splines and quadratic polynomials to the surface instead of the Gaussian kernel method. Wang et al. [22] computed two global curvatures at each voxel by integrating each of the two principal curvatures along the principal directions. Bogoni et al. [23] and Taylor et al. [24] proposed proprietary CAD algorithms for polyp detection. Pilkinton et al. [25] smoothed the image data with edge-preserving filters. They found that the smoothing did not result in a statistically significant increase in sensitivity. Gokturk et al. [20] designed a method based on the observation that the bounding surfaces of polyps are usually not exact spheres but are often complex surfaces composed of small, approximately spherical, patches. All of the above are first phase CAD methods.

The second phase of the CAD algorithms attempts to reduce the false positive (FP) identified by the first phase. In most of the methods above, curvature and curvature-based measures are the features most commonly used for classification. In particular, some $[12,13,14,15,18$, and 28] built explicit models with different degrees of complexity for the ranges of curvatures observed in colonic polyps and folds, and occasionally in the colon wall itself. However, whereas folds and polyps are modelled through highly sophisticated schemes, the colon wall is either altogether omitted or simply mentioned as region of low curvature. Gokturk et al. [20] used a support vector machine. They obtained candidate subvolumes from the first CAD phase, and then computed random triples of orthogonal cross-sections as feature vectors of the subvolumes. The subvolumes are classified using the support vector machine. Acar et al. [26] also used the displacement of edges in sequential crosssections to reduce False Positives. 

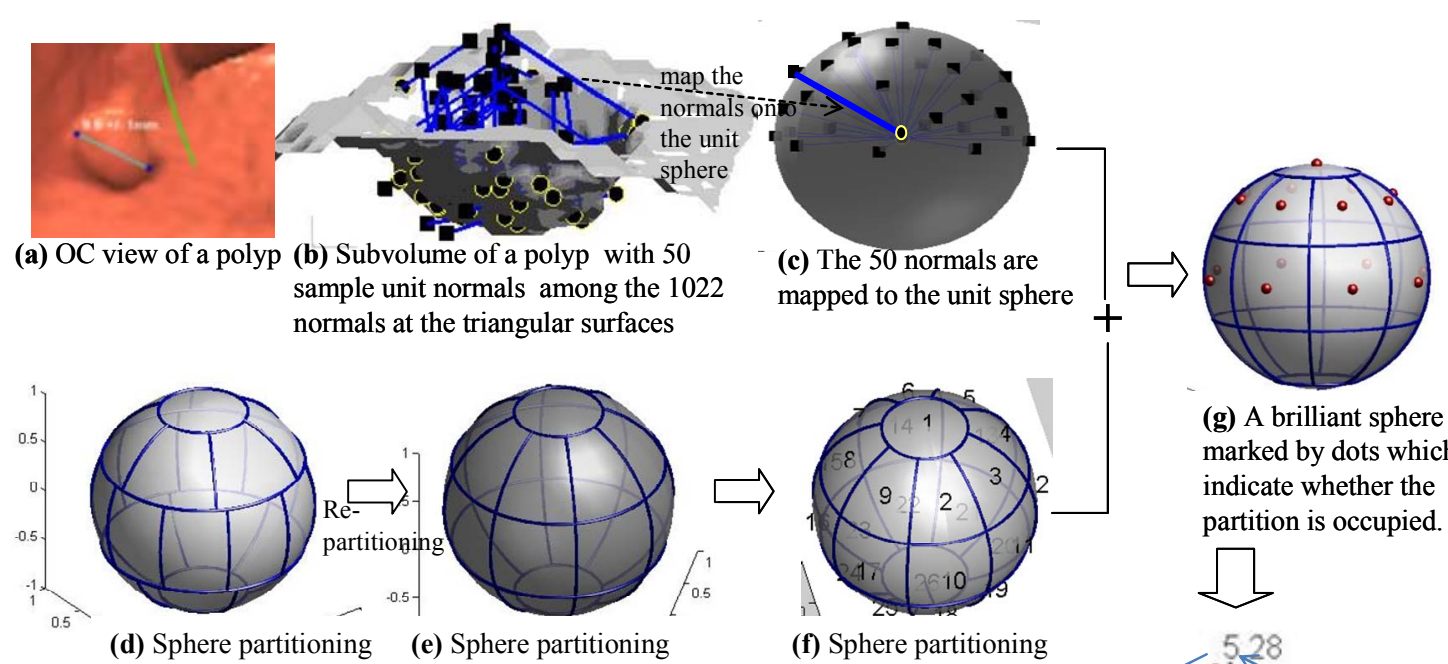

(g) A brilliant sphere is marked by dots which indicate whether the partition is occupied.

(d) Sphere partitioning

24 equal areas

(e) Sphere partitioning

26 areas based on $\theta$ and $\phi$

(f) Sphere partitioning labelling for each region

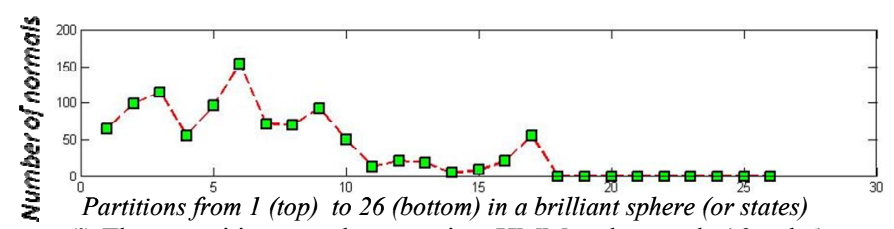

(i) These partitions are the states in a HMM and normals $(\theta$ and $\phi)$ are the observations sequences
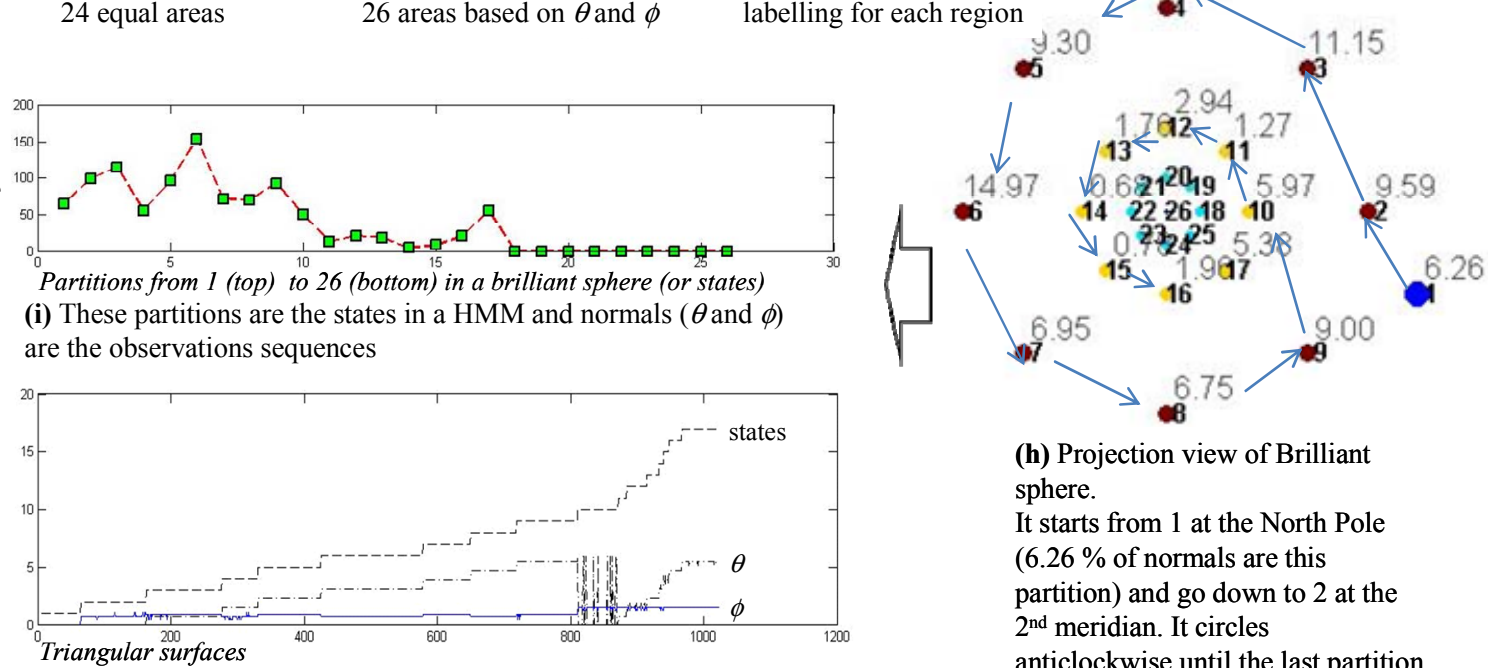

(h) Projection view of Brilliant sphere.

It starts from 1 at the North Pole (6.26\% of normals are this partition) and go down to 2 at the $2^{\text {nd }}$ meridian. It circles anticlockwise until the last partition

(j) The $\mathrm{x}$-axis represents the triangular surfaces and the normals $(\phi, \theta)$ plotted for each surface and each normal is corresponded to the states 1 9 at the meridian, and go down to 10 at the third meridian, so on. to 26 .

Figure 1. The brilliant sphere

\section{Background}

Most of the previously proposed methods assumed the shape of a polyp is close to a spherical cap. This assumption often leads to incorrect classification of large polyps as folds since the shape is a long ellipsoid. The shapes of polyp are protruding bulbous and elliptical formations and they vary in shape and size, but all shapes are limited to being a cap-like shape.

The recognition of these shapes is often performed by methods related to the Hough transform, but this method quickly leads to high dimensions and reduces efficiency. The extended Gaussian image (EGI) [27] can overcome the drawback of the Hough transform. The basic ideas of the method for different shapes are as follows:

In a flat plane every point maps to the same point on the unit sphere.
An elliptic paraboloid maps to either the upper or lower hemisphere of the unit sphere.

A sphere simply maps to cover all over the unit sphere.

However, both the Hough transform and EGI methods are unable to distinguish between folds and the polyps because the methods fail to grab the local property and the global properties at the same time. So the methods are not flexible when exposed to the various cap shapes and sizes of the folds and the polyps. In addition, there is no clear concept to enable interpretation of the EGI for such complicated shapes.

There is one well-known and certain characteristic for the shape of a polyp. It is a cap-like shape, and it contains a top (or roof) and walls. The top and walls are well connected to each other, meaning that there are no holes between them. If the object is a cube-like cap, the object has 5 parts (the roof and four walls). Hence, the normals are given as: 


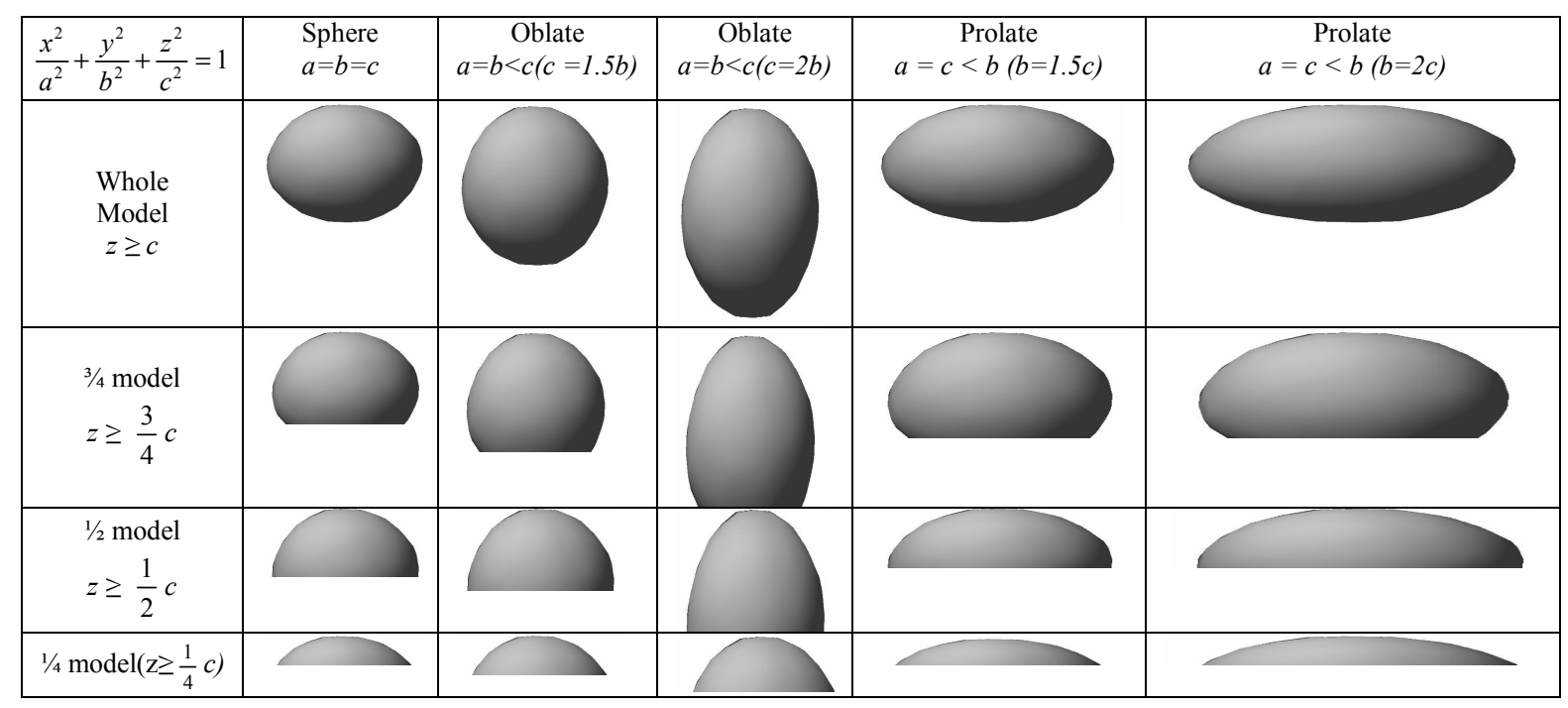

Figure 2. Spheroid models for polyps

$$
\bigcup\left\{\left(\theta_{270}, \varphi_{0}\right),\left(\theta_{90}, \varphi_{0}\right),\left(\theta_{90}, \varphi_{90}\right),\left(\theta_{90}, \varphi_{180}\right),\left(\theta_{90}, \varphi_{270}\right)\right\}
$$

where the normals are represented by the directions in spherical polar coordinates $\left(\phi_{n}, \theta_{m}\right)$, when $\mathrm{m}$ and $\mathrm{n}$ are the degree in 3D. For example, the normals from the roof are $\varphi=180^{\circ}$ and $\theta=0^{\circ}$ and the normals from the south wall are $\varphi=0^{\circ}$ and $\theta=270^{\circ}$. Because this object is a cube-like cap, the area of each side consist of a similar number of triangular surfaces, so the portion of the number of normals of one side against the number of normals of whole surface is $1 / 5$, and this portion can be obtained for other sides as well. Therefore, the sequence of normals $(\phi, \theta)$ and the number of normals for each side can define the shape. We call these properties glocal properties since the properties grab both the global information and the local information of the object. However, the polyp is not as simple a shape as was used for this example. In the domain of interest its shape is represented by the spheroid models of Figure 2, so we use a unit sphere to represent the glocal information of the polyp. We refer to this sphere as a brilliant sphere since the sphere grabs the glocal information. The brilliant sphere is shown in Figure 1.

\section{Materials}

An evaluation of the proposed procedure was performed using the subvolumes of the polyp candidates extracted from a public database (WRAMC). This database has ground truths which were marked by expert radiologists and confirmed by optical colonoscopy. The complete WRAMC database comprises many images, but unfortunately the database ground truth is provided as a distance from the rectum along the colon centerline only, and the precise location of polyps in this dataset must still be determined by an expert. The text annotation of the database might indicate that there is a polyp at the splenic flexure; however this part sometimes is not clearly visible.

It is therefore very difficult to use this database and evaluate the algorithm without knowing the exact locations of the polyps. This means it is not accurate in establishing the false positive and the false negative rates.

Therefore, we first use our proposed system to produce initial polyp candidates, and then these candidates are manually classified into the polyp database and the non-polyp database by two experienced computer scientists who have worked in medical imaging for many years. The initial candidate generation system is based on the similar concept of the surface normal overlap (SNO: Paik et al. [17]) and Kiss et al. [19]. Surface normal methods exploit the fact that normals to the colonic surface intersect with neighboring normals depending on the curvature features of the colon. The hypothesis of these kinds of methods is that polyps have a high normal intersection incidence due to their spherical nature. However, the surface of the colon is very irregular, including the smooth colonic wall, haustral folds, and polyps. Therefore, these methods have a problem creating good quality polyp candidates as the polyp candidates possibly include other close, or far, colon structures. Our system uses lengthened normals, so the algorithm detects the converging area of the normals by the detection of dense endpoints of the lengthened normals. We used various lengths of normals for the detection of various sizes of polyps. Bigger polyps can be detected by long lengthened normals, and smaller polyps can be detected by short lengthened normals, so the algorithm increases sensitivity but also increases the number of false negatives. 
The polyp database includes 43 polyps with sizes ranging from $3 \mathrm{~mm}$ to $24 \mathrm{~mm}$ and polyps are not double counted from supine and prone images. The non-polyp dataset includes 1000 subvolumes and some of these are duplicated from supine and prone images. Some of the subvolumes were duplicated from the same location in an image but extracted by the different parameters of the system.

\section{Methods}

\subsection{Mapping between normals of candidates surface and the unit sphere}

The unit normals $(\phi, \theta)$ are obtained from each point $P_{0}(x, y, z)$ on the candidate surface and these normals are mapped onto the unit sphere by moving its tail to the center of the unit sphere and its head to the surface of the unit sphere (see Figure 1.b and 1.c].

\subsection{Partitioning of the unit sphere}

The unit sphere is divided into partitions. There is a method to partition the unit sphere into equal surface areas [29], but we are more concerned about the directions rather than the surface areas. As Figure 1.c shows, 24 equal areas on the unit sphere are a bit irregular to catch the orientations of the normals between meridians. Therefore, we re-partition the sphere into 26 regions. We define $\theta$ to be the azimuthal angle in the $x y$-plane from the $x$-axis, with $0 \leq \theta \leq 2 \pi$, and define $\phi$ to be the polar angle from the $\mathrm{z}$-axis, with $0 \leq \phi \leq \pi$. The polar angle is divided into 5 meridians, and the azimuthal angle is divided into 8 regions at the $2^{\text {nd }}, 3^{\text {rd }}$ and $4^{\text {th }}$ meridian. The $1^{\text {st }}$ and $5^{\text {th }}$ meridian include the North Pole and the South Pole, and each meridian is one region. Therefore, the unit sphere is divided into 26 regions as shown in Figure 1.e. These partitions are labeled 1 to 26 running from the North Pole to the South Pole (see Figure 1.f).

\subsection{Brilliant Sphere}

The unit sphere with the surface normals of the candidate is combined with the partitioned sphere, and this sphere is called the brilliant sphere. Each partition of the brilliant sphere contains the number of normals of the surfaces of the candidate. If the portion of the number is greater than a chosen threshold, the candidate includes a side with the partition's orientation. If the partition does not have any normals, the candidate does not include a side against the directions in the partition. As Figure 1.g shows, the red small dots in the partitions represent that the partitions are occupied by a certain number of normals.

From these occupied partitions, the observation sequences $(O)$ are obtained. The reading algorithm always starts from the first partition (labeled by 1 ) and reads all normals $(\phi, \theta)$. If this partition is occupied, it comes down to second partition (labeled by 2) at the second meridian and then reads the normals. If this partitions is occupied it circles anti-clockwise to read the normals from the occupied partitions.

The observation sequences $(O)$ are given by:

$O=\bigcup_{i=1}^{26} \bigcup_{j=1}^{n}\left\{\phi_{i j}, \theta_{i j}\right\}=\left\{\left(\phi_{11}, \theta_{11}\right),\left(\phi_{12}, \theta_{12}\right) \ldots,\left(\phi_{26 \mathrm{n}}, \theta_{26 \mathrm{n}}\right)\right\}$

where $n$ is the number of normals at $i^{\text {th }}$ partition. The observation sequence will be classified by Hidden Markov Models. The classification algorithm will be presented in the next section.

\subsection{Classification of the polyp candidates}

\subsubsection{The model parameters estimation of HMM}

Generally, the model parameter estimation problem is how to adjust the HMM parameters so that the given set of observations, $O$, are represented by the model in the best way for the intended application. We create 20 models (as in Figure 2) that are suitable to represent various shapes and sizes of polyps. The models are based on an ellipsoid in the $x y z$ Cartesian coordinate system which is:

$\frac{x^{2}}{a^{2}}+\frac{y^{2}}{b^{2}}+\frac{z^{2}}{c^{2}}=1$

where $\mathrm{a}$ and $\mathrm{b}$ are the equatorial radii (along the $\mathrm{x}$ and $\mathrm{y}$ axes) and $\mathrm{c}$ is the polar radius (along the z-axis). There are 5 possible shapes defined by variations of the $\mathrm{a}, \mathrm{b}$, and $\mathrm{c}$ radii. If all three radii are equal, the ellipsoid is a sphere and the sphere is the one of the models (MS). If two radii are equal, the ellipsoid is a spheroid and we model four spheroids: MO1.5: $\mathrm{a}=\mathrm{b}$ $<\mathrm{c}$ with $\mathrm{c}=1.5 \mathrm{~b} ; \mathrm{MO} 2 \mathrm{a}=\mathrm{b}<\mathrm{c}$ with $\mathrm{c}=2 \mathrm{~b}$; MP1.5: $\mathrm{a}=\mathrm{c}<\mathrm{b}$ with $\mathrm{b}=1.5 \mathrm{c}$; and MP2: $\mathrm{a}=\mathrm{c}<\mathrm{b}$ with $\mathrm{b}=$ 2c. Based on these five models, four models are formed for each model. They are MS, MO1.5, MO2, MP1.5, MP2: whole shape ( $\mathrm{z} \geq \mathrm{c}), \mathrm{MS}^{3} / 4, \mathrm{MO} 1.53 / 4$, $\mathrm{MO} 2 \frac{3}{4}, \mathrm{MP} 1.5^{3} / 4, \mathrm{MP} 2 \frac{3}{4}$ : $3 / 4$ of the whole shape $(\mathrm{z} \geq$ 3/4c), MS $1 / 2$, MO1.51/2, MO21/2, MP1.51/2, MP21/2: half of the whole shape $(\mathrm{z} \geq 1 / 2 \mathrm{c})$, and $\mathrm{MS} 1 / 4, \mathrm{MO} 1.51 / 4$, $\mathrm{MO} 2 \frac{1}{4}, \mathrm{MP} 1.5 \frac{1}{4}, \mathrm{MP} 2 \frac{1}{4}$ : a quarter of the whole shape $(z \geq 1 / 4 c)$. For each model, the normals are calculated and map onto the brilliant sphere, then the observation sequences for each state are obtained. The mean and variance estimators are calculated to compute the parameters of the Gaussian density associated with each state. The transition matrix is also determined using the Baum-Welch algorithm. 


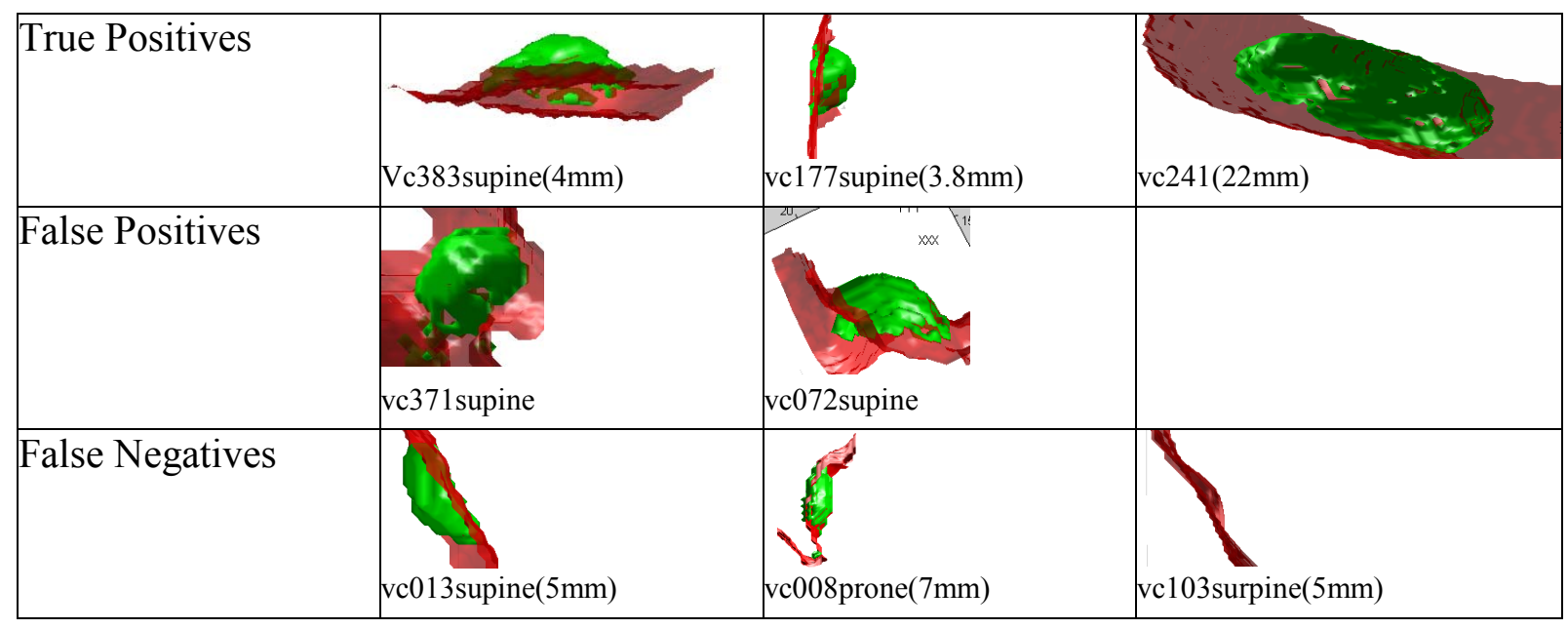

Figure 3. Examples of True Positives, False Positives, False Negatives

\subsubsection{Classifications}

The likelihood of an observation sequence $O=\left\{\mathrm{x}_{1}\right.$, $\left.\mathrm{x}_{2}, \ldots, \mathrm{x}_{\mathrm{T}}\right\}$ with respect to a HMM with parameters $\Theta$ is calculated as follows:

$$
p(X \mid \Theta)=\sum_{\text {everypossibleQ }} p(X, Y \mid \Theta)
$$

This is the sum of the joint likelihoods of the sequence over all possible state sequences allowed by the models. If any of models gives the highest likelihood to the polyp candidate, and the highest likelihood is greater than some threshold, then the candidate is assumed to be a polyp with a shape similar to that of the model.

Each polyp candidate is a sequence of spherical polar coordinates $O=\left\{\left(\phi_{1}, \theta_{1}\right),\left(\phi_{2}, \theta_{2}\right), \ldots,\left(\phi_{\mathrm{n}}, \theta_{\mathrm{n}}\right)\right\}$ where n is the length of the sequence and also the number of the triangular surface of the subvolume. These coordinates are mapped onto the brilliant sphere and a sequence of states is produced. The top of the polyp should be mapped to the North Pole of the brilliant sphere. Therefore, we translate all mapped coordinates on the brilliant sphere to match the mean coordinate to the North Pole of the sphere. Then, the log-likelihood of the sequence is computed based on our 20 HMMs using the forward and backward algorithm. If the highest log-likelihood among the results is greater than our selected threshold, the candidate is selected as a polyp. In our system, we used the Gaussian probability density function and a threshold of -1.5 .

\section{Experimental Results}

An evaluation of the proposed procedure was performed using the subvolumes of the polyp candidates extracted from a public database (WRAMC). We randomly selected 37 patients and the detection sensitivity was calculated according to the diameter of the polyps. The mean performance across the test sets in detecting "clinically significant" (where the diagnosis confidence is 2 or 3 ) colonic polyps are given below. 13 polyps were detected that are bigger than, or equal to, $10 \mathrm{~mm}$ in diameter. The sensitivity is $100 \%$ in this group and there are no false negative. 19 polyps were detected that are bigger than, or equal to, $6 \mathrm{~mm}$ in diameter and the sensitivity of this group is $91 \%$ with one false negative. 8 polyps were detected that are smaller than $6 \mathrm{~mm}$ in diameter and the sensitivity of this group is $67 \%$ with 2 false negatives. There are two false positives (see Figure 3.)

\section{Discussions}

This paper proposed an explicit parametric model for automated detection of polyps that is useful in the reduction of the number of polyp candidates identified that are false positives. The model captures the overall shape of the polyp and is used to derive the probability distribution of features that are relevant for polyp detection. The probability distribution also represents the glocal properties of the polyp candidates.

The proposed algorithm generated two false positives (vc371supine and vc072 supine in Figure 3). These look like polyps but their locations are not in the text annotations in the WRAMC database. This means that the algorithm has detected a shape that is similar to a polyp but the human expert has not classified it as a polyp. This needs to be investigated further with radiologists to examine the reason for their exclusion as polyp candidates. There are three false negatives and these are small and flat (see Figure 3). However, it should be noted also that it is a very challenging problem to detect polyps that are both small and flat. 
The partitioning decisions for the brilliant sphere also need to be discussed. There are many ways to equally divide the surface of a sphere. However, the brilliant sphere is based on the directions $(\phi, \theta)$ of the normals, so it is better to partition the sphere based on these directions. Another issue is the number of the partitions on the brilliant sphere. If there are too many partitions set to try and catch detailed local information, the state sequence obtained from the brilliant sphere does not truly represent the object as the global information of the polyp is too spread out. If a small number of partitions are set on the brilliant sphere to capture the global information of the object, it loses a lot of local information. We empirically select 26 partitions. There is one region around the North Pole, one around the South Pole, and between them we divide the sphere into 3 meridians. The middle 3 layers, which are parallel to the $x y$ plan, could be divided into 4 directions such as (positive $x$, positive $y$ ), (positive $x$, negative $y$ ), (negative $x$, positive $y$ ), and (negative $x$, negative y). However, this dividing is only useful for cubic shapes. Therefore, we divide each of the above 4 directions into 2 directions, so the partitions capture the information of flat shapes, and of curved shapes.

The HMM is often used in finding patterns which appear over a space of time, however we only use the HMM to find patterns which appear over the partitioning unit sphere (brilliant sphere). We adopt the Hidden Markov Model (HMM) to utilize the Markov property of our classification method, which is the probability of a given state coming up next $\operatorname{PR}\left(\mathrm{x}_{\mathrm{t}}=\mathrm{S}_{\mathrm{i}}\right)$, and this may depend on the history prior to $t-1$. This property allows our algorithm to detect any irregular cap shaped polyps and also various sizes of polyps.

\section{References}

[1] A guide for general practitioners, (2006) Clinical Practice Guidelines for the Prevention, Early Detection and Management of Colorectal Cancer, http://www.nhmrc.gov.au/publications/synopses/cp106/c p106divided.htm

[2] Ferlay J. Bray F. Pisani P. and Parkin D.M. GLOBOCAN 2002: Cancer incidence, mortality and prevalence worldwide. Technical report, IARC CancerBase No. 5. version 2.0 IARCPress, Lyon, France, 2004, http://www-dep.iarc.fr/

[3] ACS: http://www.cancer.org

[4] Petty T.L. (2000): Screening strategies for early detection of lung cancer: The time is nsw, JAMA, 284:1977-1980

[5] Bulman W. (2004): Screening for Colon Cancer: When Early Detection Can Mean a Cure, http://www.dentalplans.com/Dental-HealthArticles/Screening-for-Colon-Cancer-When-EarlyDetection-Can-Mean-a-Cure.asp

[6] Zheng s, Liu XY, ding KF, Wang LB, Qiu PL, ding XF, et al. Reduction of the incidence and mortality of rectal cancer by polypectomy: a prospective cohort study in Haining County, World J Gastroenterol, 8:488-492, 2002
[7] Kim DH, Pickhardt PJ, Taylor AJ, et al. CT colonography versus colonoscopy for the detection of advanced neoplasia. N Engl J Med. 357(14):1403-1412, 2007

[8] RSNA 2005 News (2005): Virtual Colonoscopy Performance Enhanced by Computer Aided Detection, http://www.rsna.org/rsna/media/pr2005/virtual colonosc opy.cfm

[9] P. Target lesion: The radiologist's perspective, In sixth International Symposium on Virtual colonoscopy, 60-62, Boston., MA, 2005

[10] RSNA 2001 News (2001): Colon Cancer Screening Improved with Computer as a 'Second Set of Eyes',

[11] Vining D.J., Ge Y., Ahn D.K. and Stelts D.R. (1999): Virtual Colonoscopy with Computer-Assisted Polyp Detection. Computer-Aided Diagnosis in Medical Imaging, 445-452

[12] Summers R.M, Beaulieu C.F., Pusanik L.M., Malley J.D., Jeffrey Jr. R.B., Glazer D.I. and Napel S. (2000): Automated polyp detector for CT colonography: Feasibility study, Radiology, 216:284-290

[13] Summers R.M., Johnson C.D., Pusanik L.M., Malley J.D., Youssef A.M. and Reed J.E. (2001): Automated polyp detection at CT colonography: Feasibility assessment in a human population, Radiology, 219:12611272

[14] Yoshida H. and Nappi J. (2001): Three-dimensional Computer-aided diagnosis Scheme for detection of Colonic Polyps, IEEE Transactions on Medical Imaging, 20:1261-1274

[15] Yoshida H., Masutani Y., MacEneaney P., Rubin D.T. and Dachman A.H. (2002): Computerized Detection of Colonic Polyps at CT Colonography on the Basis of Volumetric Features: Pilot Study, Radilogy, 222:327-336

[16] Nappi J. and Yoshida H. (2002): Automated Detection of Polyps with CT Colonography: Evaluation of Volumetric Features for Reduction of False-Positive Findings, Academic Radiology, 9:386-397

[17] Paik D.S., Beauliey C.F., Rubin G.D., Acar B., Jeffrey Jr. R.B., Yee J., Dey J. and Napel S. (2004), Surface Normal Overlap: A Computer-Aided Detection Algorithm with Application to Colonic Polyps and Lung Nodules in Helical CT, IEEE Transactions on Medical Imaging, 23(6):661-675

[18] Vos M.F., Serlie I.W.O., Gelder Van R.E., Post F.H., Truyen R., Gerritsen F.A., Stoker J., and Vossepoel A.M., A new visualization method for virtual colonoscopy., In medical image Computing and Computer-assisted Intervention, 645-654, 2001

[19] Kiss G., Cleynenbreugel J.V., Thomeer M., Suetens P. and Marchal G. (2002): Computer-Aided Diagnosis in Virtual Colonography via Combination of Surface Normal and Sphere Fitting Methods, European Radiology, 12:77-81

[20] Gokturk S.B., Tomasi B., Acar B., Beaulieu C.F., Paik D.S, Jeffrey Jr. R.B., Yee J. and Napel S. (2001): A Statistical 3-D Pattern Processing Method for ComputerAided Detection of Polyps in CT Colonography, IEEE Transactions on Medical Imaging. 20:1251-1260

[21] Li J., Huang A., Yao J., Bitter I., Petrick N., Summers M.R., Pickhardt J.P. and Choi R.J. (2006), Automatic Colonic Polyp Detection using Multiobjective Evolutionary Techniques, Medical Imaging: Image Processing, Proc. of SPIE 6144, 61445E

[22] Wang Z., Liang Z., Li L., Li X., Li B. (2005): Reduction of False Positives by Internal Features for Polyp Detection in CT-based Virtual Colonoscopy, Medical Physics, 32(12):3602-3616 
[23] Bogoni L., Cathier P., Dundar M., Jerebko A., Lakare S., Liang J., Periaswamy s., Baker M.E., Macari M., (2005): Computer-Aided Detection for CT Colonography: a Tool to Address a Growing Need. British Journal of Radiology, 78(1):S57-S62

[24] Taylor S.A., Halligan S., Burling D., Roddie M.E., Honeyfield L., McQuillan J., Amin H., Dehmeshki J. (2006) Computer-Assisted Reader Software versus Expert Reviewers for Polyp Detection on CT Colonography, America Journal of Roentgenol, 186(3):696-702

[25] Pilkinton D., Bitter I., Summers R.M., Campbell S., Choi J.R., Pickhardt P.J. (2006): The Effect of EdgePreserving Image Smoothing on Automatic Colonic Polyp Detection for CT Colonography. Medical Imaging 2006: Physiology, Function, and Structure from Medical Images, 6143:335-338
[26] Acar B., Beaulieu C., Gokturk S., Tomasi C., Paik D., Jeffrey Jr.B. Yee J., Napel S. (2002) Edge displacement field based classification for improved detection of polyps in CT colonography, IEEE Trans. Med. Imag, 21(12):1461-1467

[27] Horn BK. (1984) Extended Gaussian Images, Image Understanding Workshop, Defense Advanced Research Projects Agency, Science Appkications International Corp., 72-89

[28] M. Park, J.S. Jin, R. Hoffstetter, X. Min, and B.H. Kang, (2008), Automatic Colonic Polyp Detection by the Mapping to a Regional Unit Sphere, 2008 International Conference on Multimedia and Ubiquitous Engnieering, 144-149

[29] Paul Leopardi, (2006) A partition of the unit sphere into regions of equal area and small diameter, Electronic Transactions on Numerical Analysis, 25:309-327.

[30] Smith A.R., Cokkinides V. and Eyre J.H., (2006) American cancer society guidelines for the early detection of cancer, CA Cancer J. Clin., 569(1):11-25 